\title{
DEVELOPMENT OF PRELOADING AND PRECOOLING SYSTEMS FOR A SINGLE-TURN IGNITION TOKAMAK
}

\author{
G. W. Brunson, W. D. Booth, R. Carrera, and W. F. Weldon \\ Center for Electromechanics \\ The University of Texas at Austin \\ 10100 Burnet Rd., Bldg. 133 \\ Austin. Texas 78758-4496 \\ (512) 471-4496
}

\begin{abstract}
A basic requisite of the Fusion Ignition Experiment (IGNITEX $)^{1}$ is the production of a high $(20 \mathrm{~T})$ toroidal field (TF) by a single turn coil. The proposed high-field technology uses precooling and preloading systems. The Ignition Technology Demonstration (ITD) program, designed to produce $20 \mathrm{~T}$ on axis in a 0.06 scale prototype TF coil, utilizes a preloading structure and a precooling system. The preloading structure is a hydraulic press built around the TF coil, capable of a force of 1.1 Mlb (4.9 MN) and a stroke of 0.5 in. $(1.3 \mathrm{~cm})$. The precooling system is an open-top $\mathrm{LN}_{2}$ cryostat tub integrated into the preload press. The IGNITEX experiment is estimated to use a preload press with force capacity of approximately 150,000 tons (1.3 GN), and with a stroke on the order of 2 in. (5.1 $\mathrm{cm})$. Design considerations include efficient use of material, design of large scale hydraulic actuators, shielding to reduce radiation from activated material, maintenance, cost, and reliability. The precooling system design involves considerations of feedthroughs, minimal cooling time between pulses, maintenance and reliability.
\end{abstract}

\section{INTRODUCTION}

A basic feature of IGNITEX is the use of a preloaded, precooled single turn TF coil capable of $20 \mathrm{~T}$. Preloading (axially compressing) the TF coil isotropizes the electromagnetically-induced stresses during pulse, thereby lowering the Mises stress intensity. Precooling the coil prior to pulse maintains safe temperatures during pulse and therefore preserves the coil's material properties. The power supply for this coil is an array of homopolar generators (HPGs). To demonstrate the technology of a preloaded, precooled TF coil pulsed by homopolar generators, the ITD program at the Center for Electromechanics at The University of Texas at Austin (CEM-UT) has built and is testing a 0.06 scale TF coil prototype. This coil is pulsed by CEM-UT's 60 MJ Balcones HPG power supply. In this paper, the ITD preloading and precooling systems are described. With these systems as a base, the conceptual design of the IGNITEX preloading and precooling systems is also presented.

\section{ITD PRELOAD STRUCTURE}

As part of the ITD program, a hydraulic press has been designed and built which is capable of exerting up to 550 tons ( $4.9 \mathrm{MN}$ ) of axial preload on the 0.06 scale prototype field coil. This press can produce up to $69 \mathrm{ksi}(480 \mathrm{MPa}$ ) of compression on the TF coil's inner throat, although the initial ITD design only requires $50 \mathrm{ksi}(340 \mathrm{MPa})$. Figures 1 and 2 show the preload press.

The ITD preload press structure consists of two monolithic plates offset by six vertical links. Both the plates and vertical links are made of heattreated AISI 4340 steel. Spherical joints between the links and plates prevent the angularly-deflecting plates from causing excessive bending moments in the links when the preload is applied. Figure 3 shows the operation of the spherical joints. The press is actuated by five hydraulic cylinders (one central cylinder and four peripheral cylinders)

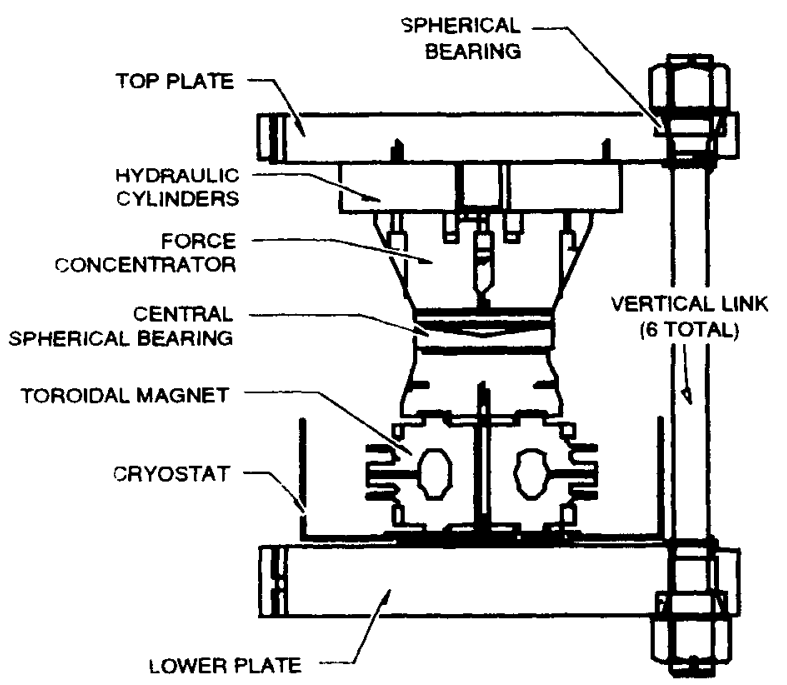

4501.0076

Figure 1. Side view of ITD preload press and 0.06 scale TF coil 


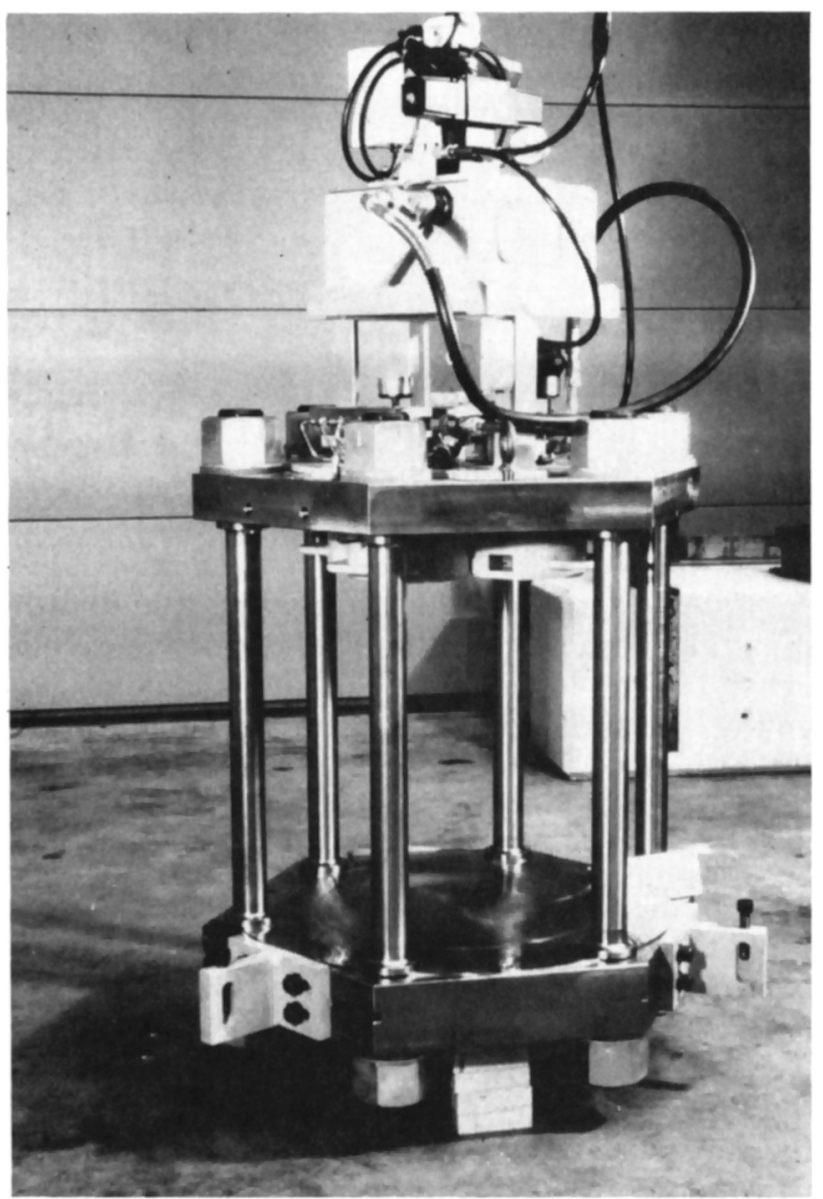

Figure 2. ITD preload press initial assembly (with out cryostat, force concentrators, or TF coil)
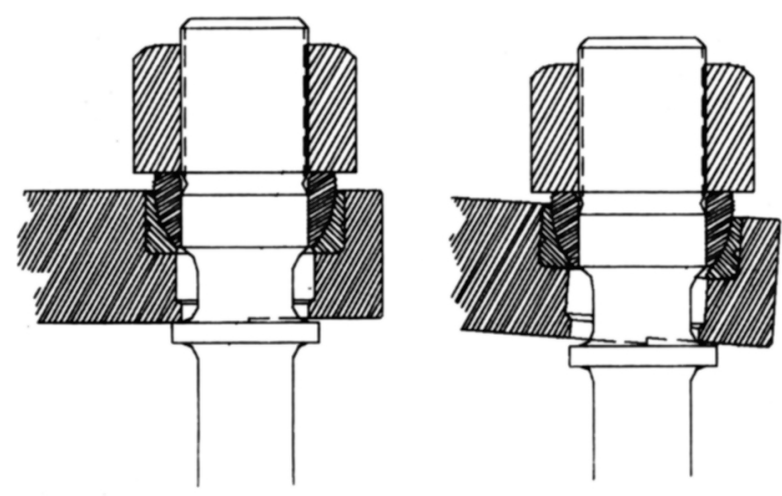

4501.0078

Figure 3. Illustration of ITD preload press pivot joint (connection between vertical links and press plates) showing components and operation attached to the underside of the top plate. The hydraulic circuit for the press is a remote controlled $10,000 \mathrm{psi}(69 \mathrm{MPa})$ system which can actuate the center cylinder only (for initial alignment of the stacked-up press components) or all cylinders in parallel. A conical steel structure concentrates the force from the cylinders onto the top of a central spherical bearing. This bearing alleviates slight angular or lateral misalignments due to the stack up of components. Composite discs (Spaulrad ${ }^{\mathrm{TM}}$ ) are placed in the stack up in direct contact with the $\mathrm{TF}$ coil in order to thermally and electrically insulate the press from the chilled TF coil.

The ITD press plates and links were designed at CEM-UT using finite-element analysis. Dtherwise, the construction of the press has relied on available technologies. The hydraulic cylinders and circuit components, spherical joints, and the rentral spherical bearing were all off-the-shelf items. The components for the press and the supporting hydraulic system had a total cost of $\$ 32,000$.

The ITD press has proved itself to be stable and easy to control. Several tests of the press to full capacity ( 550 tons) have been successfully performed. Strain measurements have been taken at critical regions, and results of these tests show that maximum stress levels in all components are acceptable and uniform. Inspection of spherical bearings has shown that these components are capable of the duty cycle required. Although the final IGNITEX preload structure will involve some more advanced technologies due to scale, the basic design principles have been verified by the ITD preload structure.

\section{ITD PRECOOLING SYSTEM}

The precooling system for the ITD TF coil includes an open-top cryostat tub (Fig. 4) which surrounds the coil. Chilling of the TF coil is achieved by filling the cryostat with $\mathrm{LN}_{2}$. Nitrogen boil-off vapor is drawn away from the experiment by an exhaust system. The buswork leading to the TF coil is designed so that the cryostat can be raised around the coil and bus, thereby preventing the need for cryogenic feedthroughs for the buswork.

The cryostat is made of 0.062 in. (1.6 mm) 304 stainless steel panels which were cut with a waterjet and welded together. The inside surface of the assembled tub was then covered with a 0.5 in. $(13 \mathrm{~mm})$ layer of urethane foam insulation. A hardened steel disc is bolted to the bottom stainless steel sheet of the cryostat and is used to transmit the preload force through the cryostat. The cryo- 


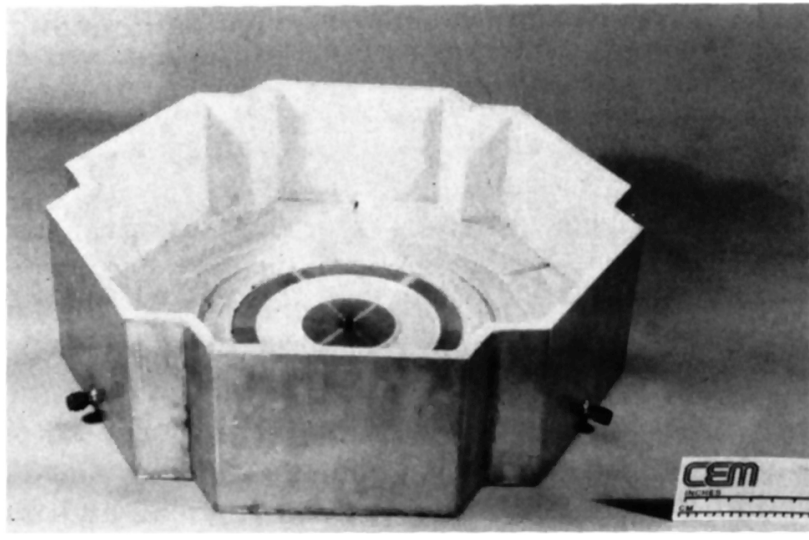

84501-108

Figure 4. ITD stainless steel, urethane foaminsulated cryostat

stat has a specialized shape with minimal clearance for the buswork and vertical links of the preload press

Mica-type band electric heaters are attached to the coaxial section of the buswork $2 \mathrm{ft}(60 \mathrm{~cm})$ from the cryostat in order to prevent condensation on the switches and HPG power supplies. Six heaters clamp to the outer diameters (ODs) of the outer coax tubes, while six others expand inside the inner diameters (IDs) of the inner coax tubes. The heaters are powered by a 480 Vac three-phase system and the 12 bus heaters are arranged to maintain a balanced delta load. The symmetry of the buswork system and magnet allows temperature control to be maintained by a single controller taking a feedback signal from the inner coax of the \#1 bus. While maintaining the ITD TF coil in the fully chilled condition, the time averaged heating load for all buswork is approximately $6 \mathrm{~kW}$.

$\mathrm{LN}_{2}$ is delivered to the cryostat from a dewar outside the CEM-UT laboratory through a main supply line which splits into three delivery lines at the cryostat. The three delivery lines tap into the cryostat through welded fittings which direct the flow of $\mathrm{LN}_{2}$ radially inward along the lower face of the TF coil. The $\mathrm{LN}_{2}$ flow is enabled by a solenoid valve located at the dewar. A commercial controller maintains the $\mathrm{LN}_{2}$ level in the cryostat by sensing the liquid level (with a capacitive probe) and controlling a solenoid valve at the dewar.

\section{IGNITEX PRELOAD STRUCTURE}

The preload stress (50 ksi or $340 \mathrm{MPa}$ ) used in the prototype TF coil will be necessary in the full size IGNITEX TF coil. Since the throat area of the
IGNITEX coil will be larger than that of the prototype's by the square of the linear scaling factor, the preload force requirement will be larger than the prototype's by approximately the same factor (278). Thus, the proposed IGNITEX experiment is estimated to use a 150,000 ton $(1.3 \mathrm{GN})$ preload structure (fig. 5). No hydraulic press presently exists with such a capacity. The largest hydraulic presses rurrently operating in the U.S. are two 50,000 ton $(440 \mathrm{MN})$ forging presses. The largest presses in the world are two forging presses in the Soviet Union capable of 82,500 tons ( $730 \mathrm{MN}$ ) each. 2 Forging experts agree that presses up to 200,000 tons $(1.8 \mathrm{GN})$ can be built with current technology, although the construction of such presses has been hard to justify to date. 3

Some important differences exist between the IGNITEX preload structure and conventional forging presses. The preload press requires much less stroke and ram speed than forging presses. Also, the preload structure does not require an accurately guided ram. The shape of the TF coil necessitates a circular bed for the preload press; this contrasts with the rectangular bed of most forging presses. These characteristics make the design and operation of the IGNITEX preload structure simpler than that of large-capacity forging presses. One press-building firm (Siempelkamp) has expressed interest in the design and construction of the IGNITEX press given the estimated budget ( $\$ 11.5 \mathrm{M}$ ); this same company has built forging presses of 66,000 ton $(590 \mathrm{MN})$ capacity and has prepared designs for larger presses.

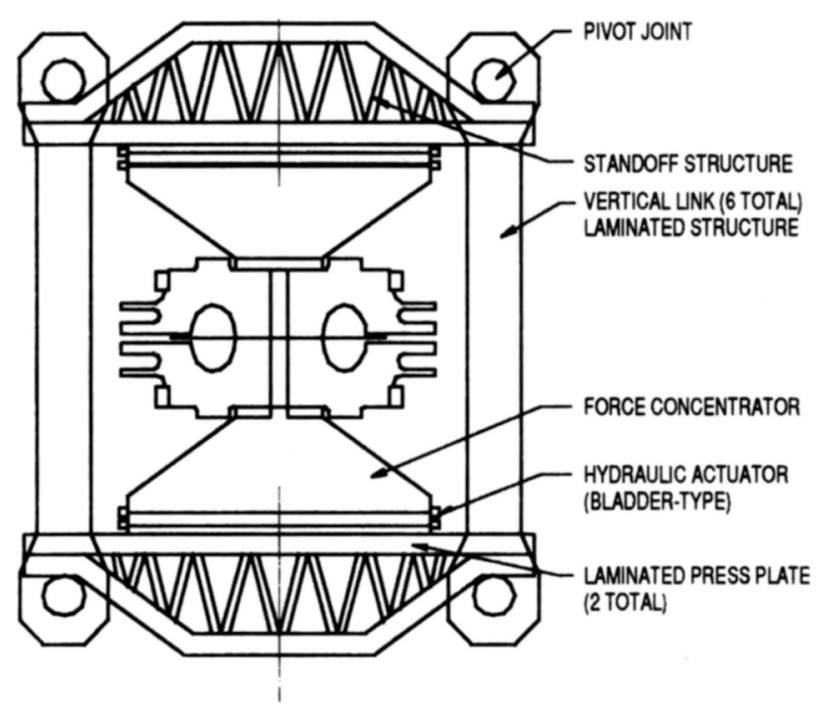

4501.0077

Figure 5. Conceptual drawing of IGNITEX preload press 
A preliminary conceptual design of the IGNITEX preload press is presented here. The preload press upper and lower plates will appear hexagonal when viewed from above. The plates will be built from bent and welded laminations. The profile of each plate will include a thick crosssection in the center (where the highest bending stresses are) with material distribution optimized for a high moment of inertia. A standoff structure within each plate will take compressive loading. The edges of the plates will be of a thinner cross section to make shear connections (between the laminations) easier and to give more room for the vertical link terminations. Conical force concentrators (also laminated) will concentrate the preload force onto Spaulrad ${ }^{\mathrm{TM}}$ spacers in contact with the TF coil upper and lower faces.

Six laminated vertical links will be loaded in tension between the upper and lower plates; the six links will leave room for the buswork which feeds current to the TF and PF coils. A gap will be included between the laminations of each link to increase radial access to the tokamak. In order to reduce bending loads in the links (as in the ITD press), pivot joints are planned between the links and the top and bottom plates. The ends of the links will be pinned to allow rolling contact with the press plates.

The scale and cost constraint of the final preload press necessitate that the components be built up from laminated plate instead of using castings or forgings. The size of these components requires a material which is available in large quantities, relatively inexpensive, and easily welded. Mild steel has been chosen as the structural material for these reasons. Efficient use of steel will be a design issue because of cost and because the press material will eventually be activated by the neutron radiation emitted in IGNITEX experiments. The surfaces of steel components which face workspaces will be coated with lead sheet in order to reduce radiation from activated material between pulses.

The hydraulic system for the preload press will be small and low-powered compared to regular forging press hydraulic systems, due to the low stroke and slow actuation requirements. Redundancy of critical system components will be built into the hydraulic circuit to enhance reliability. The hydraulic fluid will be selected to minimize activation during discharges and liquid waste disposal. Provisions will be made to allow the press to rapidly release pressure after discharge, since preloading after discharge (during thermal diffusion in the TF coil) causes higher von Mises stress intensities than during peak current.

Two designs for the hydraulic actuator are considered. An array of currently available high pressure hydraulic cylinders will be examined for feasibility and reliability. An advantage of this approach is that no new technology would be required; a disadvantage would be the relative complexity and cost of the system. A low pressure $(6,000$ psi or $41 \mathrm{MPa})$ hydraulic actuator, containing no dynamic seals, is also considered. The low pressure actuator would rely on elastic flexure of a thin steel bladder between each press end plate and the corresponding (top/bottom) force concentrator. The use of bladders both above and below the TF coil allows compliance of the coil vertically within the press, thereby simplifying the structure which supports the press in the test cell. Several bladder actuators will be stacked for the sake of redundancy. The bladder actuator has the advantages of low cost and simplicity. It also permits the elimination of dynamic seals from the design. This approach will require a demonstration of the technology of a reusable bladder.

The next phase of the ITD program at CEMUT will involve the construction and testing of a 0.12 scale TF coil and a 1-GJ HPG to furnish the current pulse. A 0.12 scale prototype of the IGNITEX preload press will be utilized to preload this TF coil. Force capacity of this press will be over 2,000 tons ( $18 \mathrm{MN}$ ). Such a model will help lend feasibility to the design and will be an excellent test bed for techniques of lamination, hydraulic actuation, and design. The full-scale preload press for IGNITEX is estimated to weigh 2,400 tons $(2,200,000 \mathrm{~kg}$ ) and is expected to cost $\$ 11.5 \mathrm{M}$ (for a more detailed cost evaluation see ref. 4). 4

\section{IGNITEX PRECOOLING SYSTEM}

A $\mathrm{LN}_{2}$ cryostat will be used to chill the full size IGNITEX TF and PF magnet systems. Fiberglass panels with a layer of urethane foam insulation and flanged edges will be connected by nonmetallic fasteners to form a low-activating cryostat vessel around the TF coil and TF terminal clamps. The cryostat panels will seal around the TF busbars and will also seal against the magnet top and bottom faces. The cryostat panels will include penetrations for PF leads, fueling, vacuum, and diagnostic systems.

The $\mathrm{LN}_{2}$ requirement for initial cooling of the $\mathrm{TF}$ coil from room temperature has been calculated experimentally. Based on the cooling requirement for the 0.06 prototype TF coil, the full size IGNITEX TF coil will require 81,000 gal $(250,000$ $\mathrm{kg}$ ) of $\mathrm{LN}_{2}$ for initial chilling. The $\mathrm{LN}_{2}$ require- 
ment for chilling between full field discharges is based on the calculated pulse energy supplied to the TF coil; this amount is $10,000 \mathrm{gal}(31,000 \mathrm{~kg})$ and is the equivalent of one truck trailer load. $\mathrm{LN}_{2}$ will be stored at the IGNITEX facility aboard the same dewar trailers used for shipping. The facility will always hold at least three loaded trailers while others are involved in transport.

During chilling, $\mathrm{LN}_{2}$ will be distributed via insulated piping into the cryostat sections. Provisions will be made to actively spray and circulate the liquid against the TF coil top and bottom surfaces for high heat flux. The exhaust vapor will be actively drawn from the cryostat at several locations and vented to outside air. The distribution and exhaust systems will be designed for minimum pressure build-up within the cryostat. Buswork heaters will be used on the TF and PF leads near the HPG power supplies to prevent unwanted condensation. When full chilling has been achieved in preparation for a discharge, the cryostat will be pumped free of any remaining $\mathrm{LN}_{2}$. After discharge, the remaining activated nitrogen gas will be pumped from the cryostat while new nitrogen vapor is used as a backfill. This activated gas will be stored under pressure until decay has rendered it safe $(\approx 2 \mathrm{hr}$ ), and then released to outside air. The precooling system will be capable of pulse chilling at a $2 \mathrm{hr}$ repetition interval.

Given the duty cycle of the IGNITEX tokamak, several modes of operation for the chilling system are envisioned. These modes are: initial chilling, pulse standby, pulse chilling, and longterm standby. The initial chilling mode is that mode in which the magnet system and buswork are brought to their respective low temperatures from room temperature. During pulse standby, the device is fully chilled, the buswork is extensively chilled (for low resistive loss during pulse), and the buswork heating system is at its highest power level (due to the resultant steep temperature gradient in the buswork). Pulse chilling is that chilling which will be necessary between successive pulses; this mode will consist of bringing the device back to $\mathrm{LN}_{2}$ temperature $(77 \mathrm{~K})$ after a discharge and restoring the "pulse-standby" temperature gradient to the buswork. During long-term standby, the magnet system will remain at $77 \mathrm{~K}$ but the buswork chilling will be relaxed, thereby allowing a more gradual temperature gradient in the buswork and therefore less bus heating load. To achieve the two different standby modes, there will be a small cryostat around each TF coil bus (inside the tokamak's concrete shielding). To achieve pulse standby, the buswork cryostats will be charged with $\mathrm{LN}_{2}$.
During long-term standby, these same cryostats will be drained. Estimated cost of the precooling system is $\$ 2.3 \mathrm{M}$ (for a more detailed evaluation of this cost see ref. 4 ).

\section{CONCLUSIONS}

A relatively simple and low cost fusion ignition experiment (IGNITEX) is considered. The experiment can be realized in a single-turn tokamak capable of producing a $20 \mathrm{~T}$ field on the plasma axis. At this field level, active axial preloading of the TF coil is required. The conceptual design of the required preload structure is presented here. The demonstration of advanced preloading technologies is proposed in a 0.12 scale prototype system. The pulse length envisioned for the IGNITEX experiment $(10 \mathrm{~s})$ requires precooling of the full magnet system to $77 \mathrm{~K}$ between pulses. Design and operation of a cryogenic system capable of handling the IGNITEX requirement is described here. The basic design characteristics of preloading and precooling systems for high current, single-turn toroidal configurations have been demonstrated in the ITD program at CEM-UT. Results to date have been presented here, including successful operation of a $1.1 \mathrm{Mlb}(4.9 \mathrm{MN})$ hydraulic preload press built for the 0.06 scale TF coil prototype.

\section{ACKNOWLEDGMENTS}

Funding for the ITD program is provided by the Texas Advanced Technology Program and the Texas Atomic Energy Research Foundation.

\section{REFERENCES}

[1] M. N. ROSENBLUTH, W. F. WELDON, AND H. H. WOODSON, "Basic Design Report for the Fusion Ignition Experiment (IGNITEX)," Center for Fusion Engineering Report, March 1987.

[2] T. ALTAN AND $S$. L. SEMIATIN, "Feasibility of Using a Large Press $(80,000$ 200,000 Ton) for Manufacturing Future Components on Army Systems," U.S. Army TankAutomotive Command R\&D Center Contract No. DAAE07-83-M-R028, Final Report, July 1983.

[3] M. SFILIGOJ, "Forgers Aren't Sure They Require any Presses Exceeding 50,000 Tons," American Metal Market, Vol. 91, June 6, 1983.

[4] D. BOOTH, P. COOPER, G. BRUNSON, R. CARRERA, M. MANAVAZHI, J. QUINONES, W.A. WALLS, W.F. WELDON, AND M.D. WERST, "Cost Evaluation of the Fusion Ignition Experiment IGNITEX," Ninth Topical Meeting in the Technology of Fusion Energy, Oak Brook, IL, October 1990. 